\title{
Endovascular embolisation of Giant Ruptured Proximal Splenic Arterial Pseudoaneurysm Using Microcoils \& Glue: Case Report
}

\author{
Jawahar Rathod ${ }^{1}$, Kishor Taori ${ }^{1}$, Sachin Dhomane ${ }^{2}$, Prakash Pawar ${ }^{3}$, Devkaran Vaghasiya ${ }^{1}$, \\ Amit Disawal $^{1}$, Amrita Guha ${ }^{1}$, Kiran Naiknavare ${ }^{1}$, Rahul Agale ${ }^{1}$, Prajwleet Gour ${ }^{1}$ \\ ${ }^{1}$ Department of Radio-diagnosis, Government Medical College and Hospital, Nagpur, India \\ ${ }^{2}$ Care Hospital, Nagpur, India \\ ${ }^{3}$ Sion Medical College \& Hospital, Mumbai, India. \\ E-mail: drjawahar_12@rediffmail.com \\ Received January 10, 2011; revised April 15, 2011; accepted April 22, 2011
}

\begin{abstract}
Splenic artery aneurysms (SAA) are third most common intra-abdominal aneurysm (after aneurysms of the abdominal aorta and iliac arteries). A splenic artery aneurysm larger than $3 \mathrm{~cm}$ is even rare. Splenic artery aneurysms are fatal vascular lesions if ruptured. The incidence of rupture is increased in larger aneurysms. Transcatheter arterial embolization is an alternative method of treatment in a patient with splenic arterial aneurysm which has relative lower morbidity and mortality than surgical procedures. Here, we report a case of giant ruptured proximal splenic artery pseudoaneurysm detected by contrast enhanced computed tomography (CECT) \& referred to Interventional Radiology for Transcatheter arterial embolisation.
\end{abstract}

Keywords: Ruptured, Pseudoaneurysm, Microcoils, Glue, Pancreatitis

\section{Introduction}

Aneurysms of visceral arteries are found in less than $1 \%$ of the general population [1-4]. Splenic artery aneurysms are the most common visceral artery aneurysms, accounting for $60 \%$ to $71 \%$ [5-7], with a reported prevalence of $0.8 \%$ at arteriography [8]. SAA are more common in women than men. Pregnancy is the major risk factor for rupture. Here, we report a case of acute on chronic pancreatitits with giant ruptured proximal splenic artery pseudoaneurysm which was successfully embolised using microcoils \& cyanoacrylate glue.

\section{Case Report}

A 40-year-old male patient who was previously diagnosed as a case of acute hemorrhagic pancreatitits \& managed conservatively 6 months back at our institution, now came with h/o pain in epigastric region, hematemesis \& malena since 2 days. Laboratory tests revealed slightly raised Sr. lipase (214 U/L, normal: upto $190 \mathrm{U} / \mathrm{L})$ \& anemia (Hb: 10.5 gm\%). USG abdomen \& Computed tomography (CT) was performed, it showed changes of acute on chronic pancreatitits (Figure 1) \& a large splenic artery pseudoaneurysm in its proximal part measuring $5 \times 3.8 \mathrm{~cm}$. The aneurysm consists of peripheral thrombosed part (Figure 2).

The patient was then referred to Interventional Radiology department for emergency angio-embolisation after giving Pneumococcal (pneumovac) and H. Influenzae B vaccination. Using Seldinger's method, via right transfemoral approach, Selective angiography of the splenic artery was performed using 5 Fr SIM 1 catheter (cordis), It showed a large sessile pseudoaneurysm arising from the proximal segment of the splenic artery (Figure 3). Through SIM 1 catheter, microcatheter (Miraflex, 2.5 Fr) introduced into the splenic artery as a co-axial system, tip of the microcatheter was placed beyond the pseudoaneurysm and embolisation started using microcoils of various sizes (6 to $8 \mathrm{~mm}$ ) but all the coils (Total 8 coils) migrated at a single place of acute turn of distal splenic artery. Angiography at this stage showed patent aneurysm with embolisation of distal splenic artery. Then the decision was taken to embolise whole of the splenic artery proximal to the microcoils using Glue (N-butyl Cyanoacrylate). $6 \mathrm{ml}$ 50\% glue mixed with 


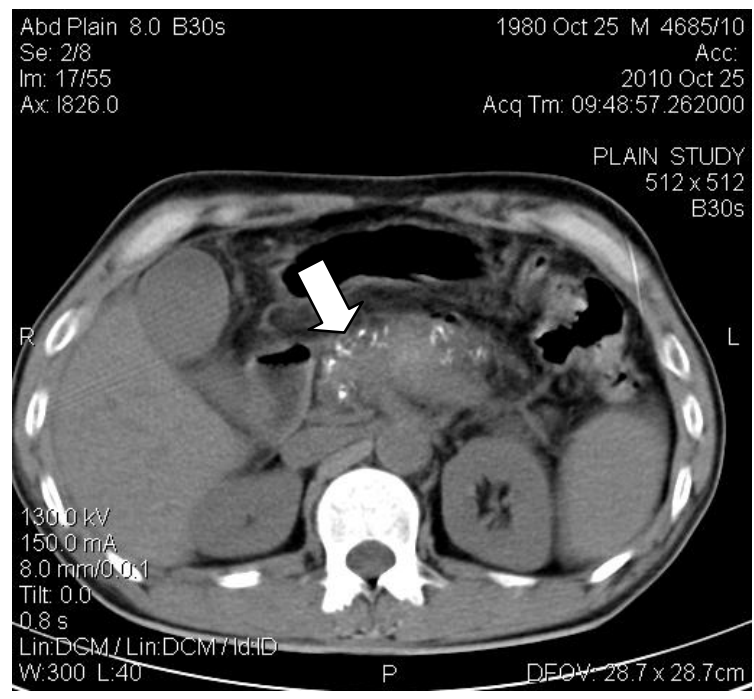

Figure 1. NECT abdomen axial cuts reveals bulky heterogenous pancreas with multiple intra-pancreatic calcifications.

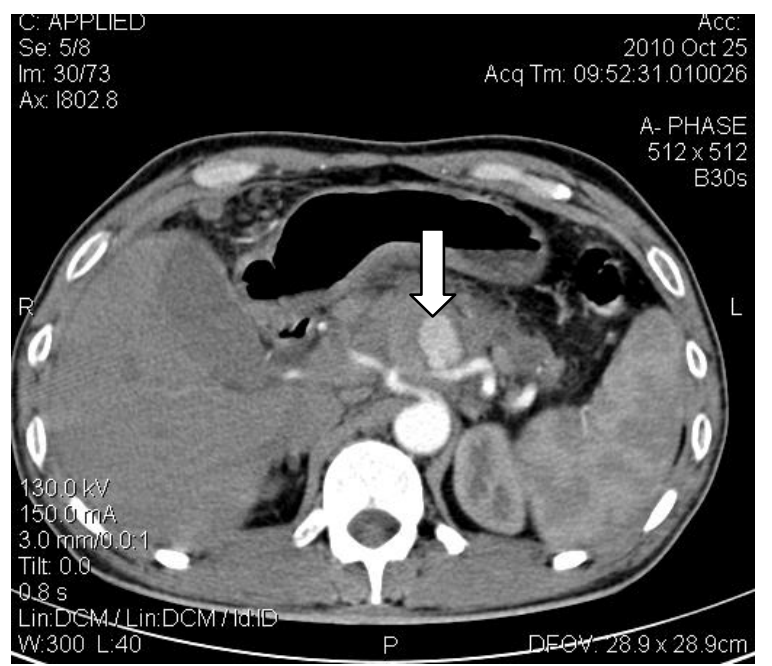

Figure 2. CECT abdomen axial cuts reveals giant saccular anteriorly placed pseudoaneurysm (peripheral thrombosed part \& central patent part) arising from proximal splenic artery.

lipiodol in equal proportion injected through the microcatheter upto the origin of the splenic artery. Bunch of microcoils in distal splenic artery prevented glue from entering into the splenic parenchyma. Post-embolization angiogram \& fluoroscopic image revealed successful embolisation of the splenic artery with non-filling of the pseudoaneurysm with microcils in the distal splenic artery \& glue cast in the proximal splenic artery. (Figures 4 and 5)

\section{Discussion}

Splenic artery aneurysms are being diagnosed more com-

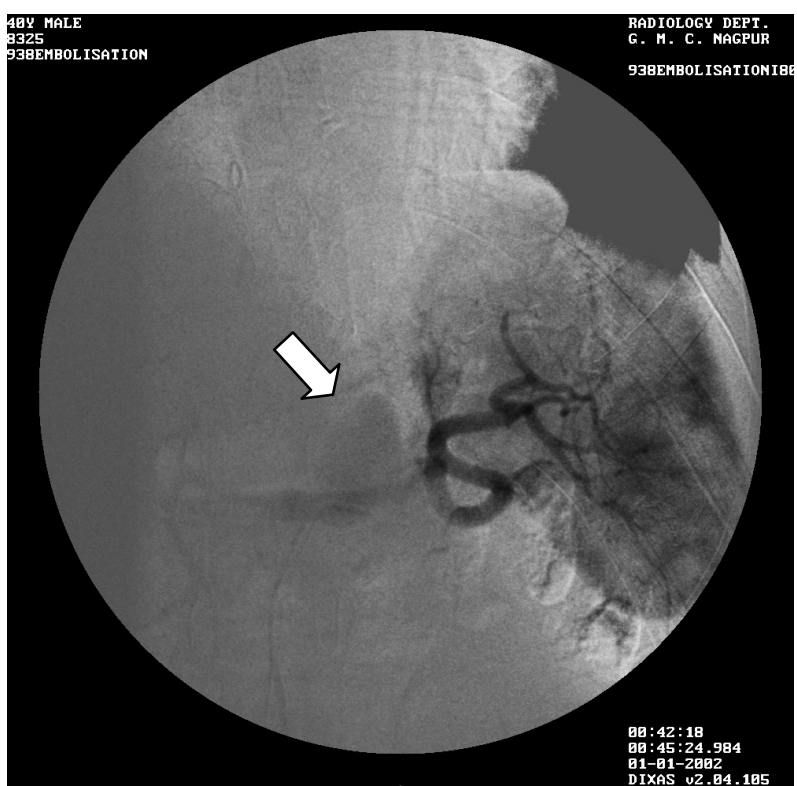

Figure 3. DSA angiogram of splenic artery showing pseudoaneurysm from proximal splenic artery

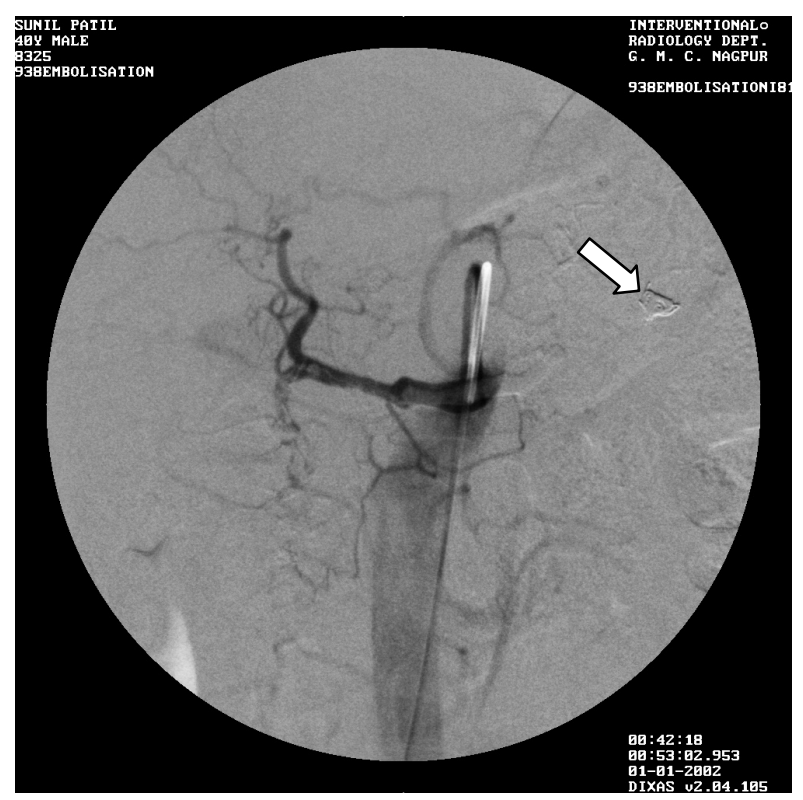

Figure 4. Post embolisation celiac angiogram showing peripheral bunch of coils with glue cast in the entire splenic artery with patent hepatic $\&$ left gastric artery.

monly than in the past, mainly because of increased availability of modern radiological imaging techniques like computed tomography and angiography $[5,6,9]$. Treatment of splenic artery aneurysm is recommended in patients with aneurysms greater than $2 \mathrm{~cm}$ [10]. Splenic artery aneurysms are found most often in multiparous women attributed to intimal hyperplasia. The prevalence of splenic artery aneurysm has increased in patients with portal hypertension and is estimated at $7 \%-20 \%$ [11]. 


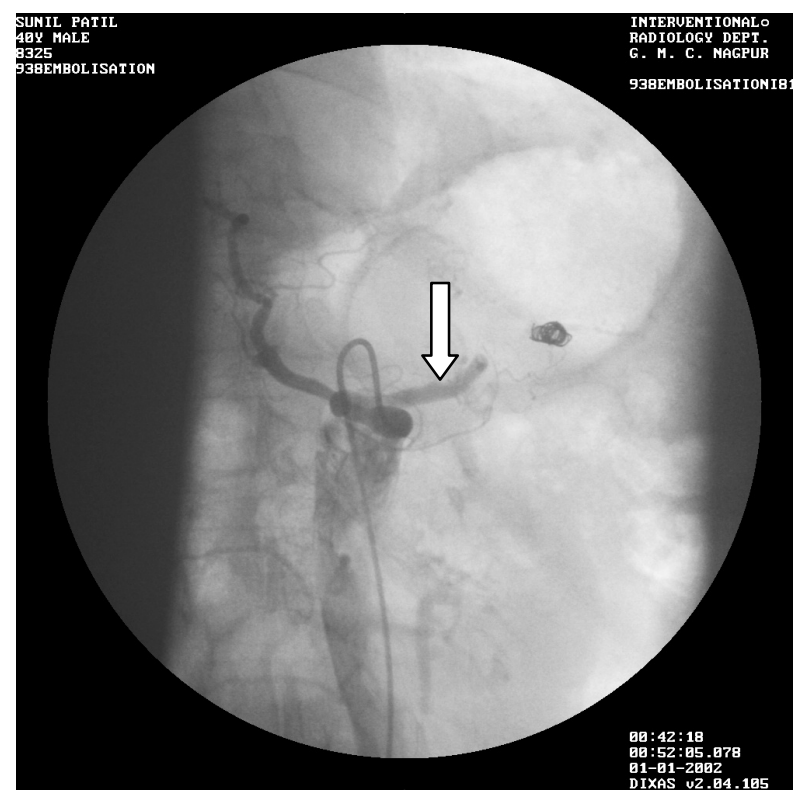

Figure 5. Post embolisation fluoroscopic image showing peripheral bunch of coils with glue cast (arrow) in the proximal splenic artery with patent hepatic \& left gastric artery.

Splenic artery pseudoaneurysm may be seen in patients with trauma \& pancreatitis. The splenic artery is the most common site for pseudoaneurysms secondary to pan creatitis [11]. Treatment of pseudoaneurysms is mandatory because risk of rupture is high $[12,13]$. These patients are often ill with severe pancreatitis and a minimally invasive form of treatment would be of value. Here we report a similar case in which the patient presented with splenic artery pseudoaneurysm which was ruptured \& presented with hematemesis \& malena.. The results of surgery for SAPAs are influenced by the patient's overall condition, and also by the anatomic location of the pseudoaneurysm. Surgical mortality is approximately $16 \%$ for pseudoaneurysms in the head of the pancreas and 50\% for lesions in the tail [12]. Other uncommon causes of splenic artery aneurysm include fibromuscular dysplasia, infection, and congenital anomaly [13]. Most aneurysms are small (2 cm in diameter), saccular, and located at a bifurcation in a middle or distal segment of the splenic artery [5,6,14]. The incidence of rupture of splenic aneurysms is reported relatively less frequently, occurring in $2 \%$ to $10 \%$ of cases, but the risk increases when the aneurysmal diameter exceeds $3 \mathrm{~cm}$, when the size increases within a relatively short period, or when the patient is pregnant $[3,4,15]$. Most splenic artery aneurysms are detected incidentally during diagnostic imaging performed for other indications. Rupture of splenic artery aneurysm is rare; however, the ruptured splenic artery aneurysm left untreated is associated with a high mortality rate [8]. In the present case report, the size of the pseudoaneurysm was more than $3 \mathrm{~cm} \&$ it was ruptured. Treatment of splenic artery aneurysm is recommended in women of childbearing age, patients undergoing liver transplantation, patients with symptomatic or expanding aneurysms, and patients with aneurysms greater than $2 \mathrm{~cm}[8,10]$. Endovascular methods are coming to the forefront for the management of splenic artery aneurysms. Splenic artery aneurysms away from the hilum can be excluded with a stent graft. Embolization of the entire splenic artery, if selective catheterization of the aneurysm cannot be performed, is an alternative option $[8,10]$. We have also performed complete embolisation of the splenic artery, distal \& proximal to the pseudoaneurysm using two different embolisation materials, one is microcoils \& another is liquid embolising material i.e. Glue for the first time. Surgery for splenic artery aneurysms is associated with a mortality rate of approximately $1 \%$, but the mortality rate is increased in patients with pancreatitis, in whom it is $16 \%$ for those with aneurysms in the pancreatic head and 50\% for those with pancreatic body aneurysms. Splenic artery aneurysms also may be treated with percutaneous interventional techniques such as transcatheter arterial embolization, placement of a covered stent to exclude the aneurysm, or percutaneous injection of coils or thrombin. Transcatheter arterial embolization is associated with significantly lower morbidity and mortality rate than surgical procedures [16,17]. Embolization of intrasplenic lesions may be performed with microcatheter-based techniques, and success rates of $80 \%-90 \%$ have been reported for percutaneous transcatheter embolization[8]. Stent-graft placement across the aneurysmal neck to exclude the aneurysm, a treatment that provides the benefit of preserving blood flow through the splenic artery has been reported $[18,19]$. In our case, we deployed the metallic coils in the splenic artery distal to the aneurysm \& injected glue so that there was permanent complete occlusion of the splenic artery \& its aneurysm with no passage of cyanoacrylate glue into the distal most splenic artery \& its branches. It provides a cost effective method to treat proximal splenic artery pseudoaneurysm. The spleen has a rich vascular collateral supply, therefore embolization is a more attractive treatment option than it would be in other visceral arteries. The occurrence of complications after endovascular treatment is uncommon. Possible complications include postembolization syndrome, transient elevation of pancreatic enzymes, splenic infarction, infection, abscess, and, rarely, rupture of a pseudoaneurysm $^{8}$. Follow up imaging with CT or Doppler ultrasonography is recommended for assessment of the adequacy of treatment. The indications that warrant surgical intervention i.e. splenectomy after splenic artery embolisation include hematologic complications and 
persistent symptoms, such as sepsis, abscess, hemorrhage, or persistent pseudocyst formation.

\section{Conclusions}

Proximal splenic arterial pseudoaneurysms can safely be embolised by this novel technique of placing appropriate microcoils distal to the pseudoaneurysm \& embolisation of proximal splenic artery using 50\% glue rather than the traditional method of embolising whole of the splenic artery with coils.

\section{References}

[1] J. M. Graham, C. H. McCollum and M. E. DeBakey, "Aneurysm of the Splanchnic Arteries," The American Journal of Surgery, Vol. 140, No. 6, 1980, pp. 797-801. doi:10.1016/0002-9610(80)90120-8

[2] M. Negita, H. Suenega, Y. Suzuki, et al, “A Case Report Ofaneurysm in the Root of Splenic Artery of the Splenomesenteric Type Treated with Reanastomosis," Nippon Geka Gakkai Zasshi, Vol. 93, No. 3, 1992, pp. 332-335.

[3] P. G. Settembrini, J. M. Jausseran, S. Roveri, et al, “Aneurysms of Anomalous Splenomesenteric Trunk: Clinical Features and Surgical Management in Two Cases,” Journal of Vascular Surgery, Vol. 24, No. 4, 1996, pp. 687692. doi:10.1016/S0741-5214(96)70085-X

[4] D. M. Rogers, J. E. Thompson, W. V. Garrett, C. M. Talkington and R. D. Patman, "Mesenteric Vascular Problems: A 26-Year Experience," Annals of Surgery, Vol. 195, No. 5, 1982, pp. 554-565. doi:10.1097/00000658-198205000-00004

[5] V. F. Trastek, P. C. Pairolero, J. W. Joyce, L. H. Hollier and P. E. Bernatz, "Splenic Artery Aneurysms," Surgery, Vol. 91, 1982, pp. 694-699.

[6] M. A. Abbas, W. M. Stone, R. J. Fowl, et al, "Splenic artery aneurysms: Two decades experience at Mayo clinic,” Annals of Vascular Surgery, Vol. 16, 2002, pp. 442449. doi:10.1007/s10016-001-0207-4

[7] K. S. Baker, J. Tisnado, S. R. Cho and M. C. Beachley, "Splanchnic Artery Aneurysms and Pseudoaneurysms: Transcatheter Embolization,” Radiology, Vol. 163, No. 1, 1987, pp. 135-139.

[8] D. C. Madoff, A. Denys, M. J. Wallace, et al, "Splenic Arterial Interventions: Anatomy, Indications, Technical Considerations, and Potential Complications," Radiographics, Vol. 25, 2005, pp. S191-S211. doi:10.1148/rg.25si055504

[9] D. J. Tessier, W. M. Stone, R. J. Fowl, et al, "Clinical Features and Management of Splenic Artery Pseudoaneurysm: Case Series and Cumulative Review of Lit- erature," Journal of Vascular Surgery, Vol. 38, No. 5, 2003, pp. 969-974. doi:10.1016/S0741-5214(03)00710-9

[10] Z. C. Schmittling and R. McLafferty, "Transcatheter Embolization of a Splenic Artery Aneurysm,” Journal of Vascular Surgery, Vol. 40, No. 5, 2004, p. 1049. doi:10.1016/j.jvs.2003.12.003

[11] M. Sato, I. Anno, M. Yamaguchi, H. Iida and K. Orii, "Splenic Artery Aneurysm of the Anomalous Splenomesenteric Trunk: Successful Treatment by Transcatheter Embolization Using Detachable Coils,” Cardiovascular and Interventional Radiology, Vol. 29, No. 3, 2005, pp. 432-434. doi:10.1007/s00270-005-0036-x

[12] C. D. Long, K. R. Bakshi, M. B. Kahn and A. B. Roberts, "Giant Splenic Artery Aneurysm," Annals of Vascular Surgery, Vol. 7, No. 5, 1993, pp. 474-478. doi:10.1007/BF02002133

[13] A. Ayalon, R. H. Wiesner, J. D. Perkins, S. Tominaga, D. H. Hayes and R. A. Krom, "Splenic Artery Aneurysms in Liver Transplant Patients,” Transplantation, Vol. 45, 1988, pp. 386-389. doi:10.1097/00007890-198802000-00028

[14] J. Golzarian, N. Nicaise, J. Deviere, et al, “Transcatheter Embolization of Pseudoaneurysms Complicating Pancreatitis," Cardiovascular and Interventional Radiology, Vol. 20, No. 6, 1997, pp. 435-440. doi:10.1007/s002709900189

[15] R. W. Bailey, T. S. Riles, R. J. Rosen and L. P. Sullivan, "Celiomesenteric Anomaly and Aneurysm: Clinical and Etiologic Features,” Journal of Vascular Surgery, Vol. 14, 1991, pp. 229-234. doi:10.1067/mva.1991.28728

[16] V. G. McDermott, R. Shlansky-Goldberg and C. Cope, "Endovascular Management of Splenic Artery Aneurysms and Pseudoaneurysms," Cardiovascular and Interventional Radiology, Vol. 17, No. 4, 1994, pp. 179-184. doi:10.1007/BF00571531

[17] R. Guillon, J. M. Garcier and A. Abergel, et al, "Management of Splenic Artery Aneurysms and False Aneurysms with Endovascular Treatment in 12 Patients," Cardiovascular and Interventional Radiology, Vol. 26, No. 3, 2003, pp. 256-260. doi:10.1007/s00270-003-1948-y

[18] A. Arepally, M. Dagli, L. V. Hofmann, H. S. Kim, M. Cooper and A. Klein, "Treatment of Splenic Artery Aneurysm with Use of a Stent-Graft," Journal of Vascular and Interventional Radiology, Vol. 13, 2002, pp. 631-633. doi:10.1016/S1051-0443(07)61659-5

[19] E. N. Brountzos, K. Vagenas, S. C. Apostolopoulou, I. Panagiotou, D. Lymberopoulou and D. A. Kelekis, "Pancreatitis-Associated Splenic Artery Pseudoaneurysm: Endovascular Treatment with Self-Expandable Stentgrafts," Cardiovascular and Interventional Radiology, Vol. 26, No. 1, 2003, pp. 88-91. doi:10.1007/s00270-002-1979-9 